\title{
Finding the Relationship Between the Biological Dagradtion Rate Constants and Some of the Calculated Physical Properties of a Number of Carcinogenic poly Aromatic Hydrocarbons
}

\author{
Rayan Basheer Mahmood \\ Zaheda Ahmed Najim \\ Chemistry Department / College of Education for Pure Science \\ University of Mosul \\ rayan.mahmood@ gmail.com \\ zahedahmed@uomosul.edu.iq. \\ DOI: $\underline{10.33899 / \text { edusi.2019.162976 }}$
}

$\begin{array}{cc}\text { Received } & \text { Accepted } \\ 28 / 11 / 2018 & 27 / 02 / 2019\end{array}$

\begin{abstract}
This study involves the theoretical calculation of binding energy (the free energy $\Delta \mathrm{G}^{\circ}$ ) of eleven of polycyclic aromatic hydrocarbons (PAHs) compounds with catechol 1,2-dioxygenase enzyme from the gram-positive Rhodococcus opacus (3HGI) enzyme that is used for degrading these types of compounds and also identifies the values of binding energy that compactable with constant values of biological degradation rate. It is noted that binding energy inversely proportional with rate constants and binding energy $(\mathrm{R}=0.951)$, as the binding energy with enzyme are increased, the degradation rate are decreased.

The study observes that binding energy values were ranging between ([-5.051] - [-7.12] $\mathrm{Kcal} / \mathrm{mol}$ ) whereas values of root mean squared deviation (RMSD) were ranging between $\left(1.12-1.71 \mathrm{~A}^{\circ}\right)$ by which accurate results have been confirmed depending on previous studies. The study also determines the amino acids that surround the compounds which are usually blinded by the hydrogen bond or hydrophobic interactions types $\pi-\pi$.

Finally, the study obtains a three - dimensional shape of compound's binding with enzyme of its more stable shape. Also obtains the shapes of distribution of the electronic cloud on the compound and amino acids that are surrounding them in which getting locations around the studied compound's molecule depending on their features of an amino acid (acidity - basic - hydrophobic). MOE (Molecular Operating Environment) is used for this step of calculation.
\end{abstract}

Keywords: Binding energy $\left(\Delta \mathrm{G}^{\circ}\right)$, Poly aromatic hydrocarbons (PAHs), Biological degradation rate constants, Amino acids, Enzyme catechol 1,2dioxygenese (3HGI). 


\section{إيجاد العلاقة بين ثوابت سرعة التحلل البايولوجي وبعض الصفات الفيزياوية المحسوبة نظرياً لعدد من المركبات الاروماتية الهياروكربونية متعددة الحلقات المسببة للسرطان}

$$
\begin{aligned}
& \text { ريان بثير محمود اهدة احمد نجم } \\
& \text { قسم الكيمياء / كلية التربية للعلوم الصرفة }
\end{aligned}
$$

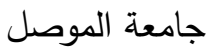

\section{zahedahmed@uomosul.edu.iq rayan.mahmood@gmail.com}

DOI: $10.33899 /$ edusi.2019.162976

$$
\begin{aligned}
& \text { القبول } \\
& 2019 \text { | } 02 \text { | } 27 \\
& \text { الاستلام } \\
& 2018 \text { / } 11 \text { / } 28
\end{aligned}
$$

\section{الخلاصة}

تضمنت هذه الدراسة حساب طاقة الارتباط (الطاقة الحرة) لأحد عشر مركباً من المركبات الهيدروكربونية متعددة الحلقات مع أنزيم كاتيكول 2,1 - ثنائي أوكسجنيز 3HGI الذي يعد أحد الأنزيمات

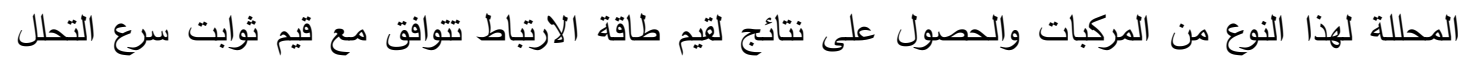
الحيوي إذ وجد بأن طاقة الارتباط تتناسب عكسياً مع ثوابت السرعة وبمعامل ارتباط (R=0.951) إذ إذ إنه بزيادة طاقة الارتباط مع الأنزيم تقل سرعة التحلل.

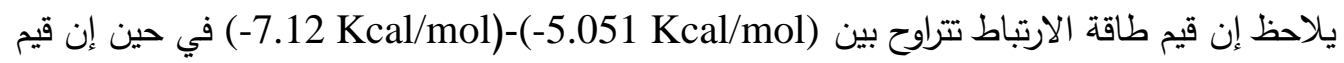

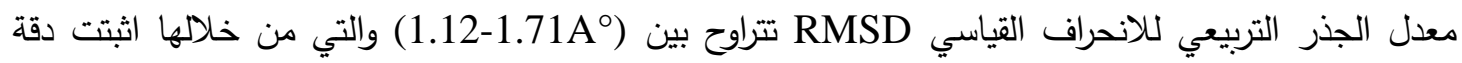
النتائج استتاداً إلى دراسات سابقة وتم ايضاً تحديد الأحماض الأمينية التي تحيط بالمركب والتي عادئ مادئ ما ترتبط

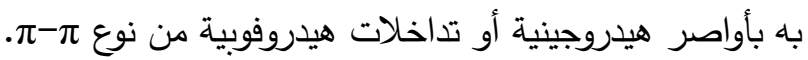
وفي النهاية تم الحصول على الثكل ثلاثي الابعاد لارتباط المركبات مع الأنزيم بهيئتها الاكثر استقراراً وكذلك الحصول على اشكال توزيع السحابة الالكترونية على المركبات والأحماض الأمينية المحيطة بها والتي تأخذ مواقعاً حول جزئة المركب المدروس استناداً إلى صفاتها كأحماض أمينية حامضية, قاعدية, هيدروفوبية

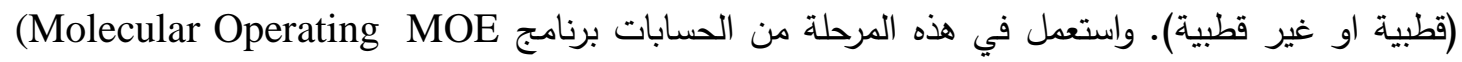
.Enviroment)

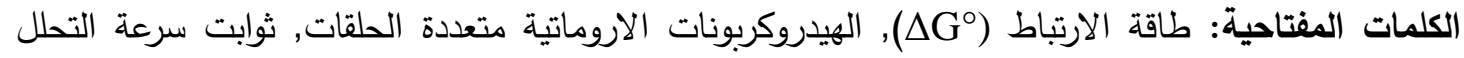
الحيوي, الأحماض الأمينية, أنزيم كاتيكول 2,1- ثائي أوكسجنيز • 


\section{Introduction}

المقدمة

1-1 1 الهيدروكربونات الاروماتية متعددة الحلقات :

هي مركبات كيميائية تتكون من حلقات اروماتية مدموجة ولا تحتوي على ذرات متغايرة ولا يوجد

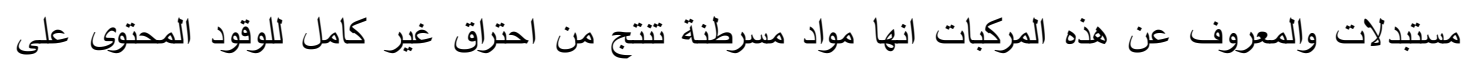

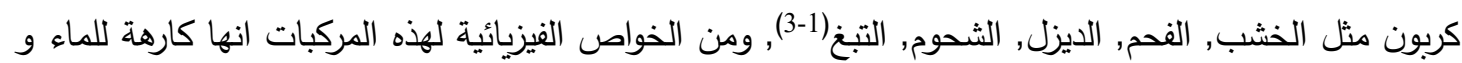

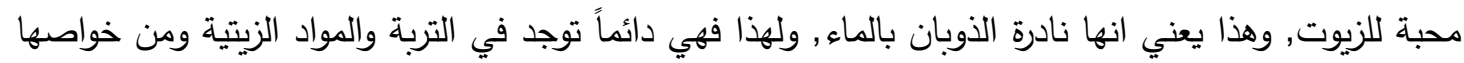

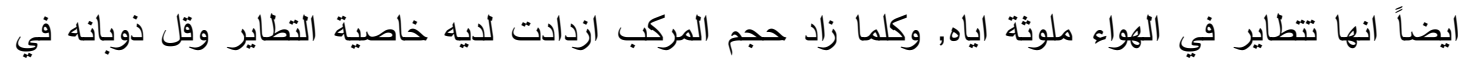
الماء (4).

\section{PAHs 1 2-2 ميكانيكية التحلل (التكسير) الحيوي لمركبات}

الأنزيمات عبارة عن مواد بايولوجية محفزة (مساعدة) تقوم وبكميات قليلة بزيادة سرعة التفاعلات

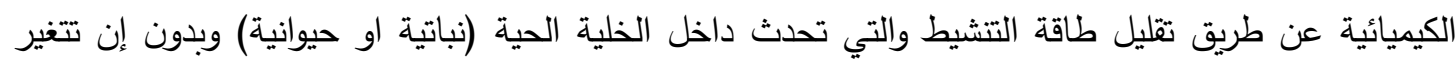
خلال هذه التفاعلات.

تحتوي جميع الأنزيمات على منطقة تسمى الموقع الفعال (Active site). إن القوى التي تربط المادة

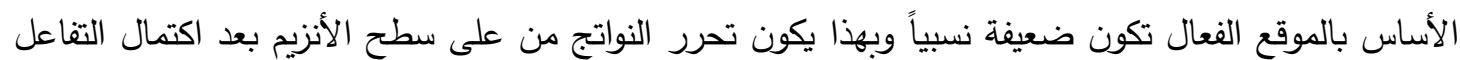

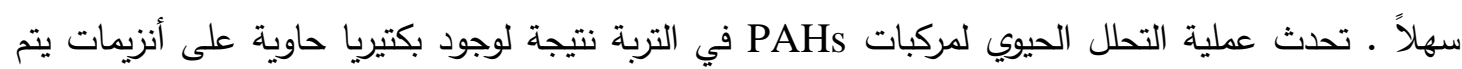

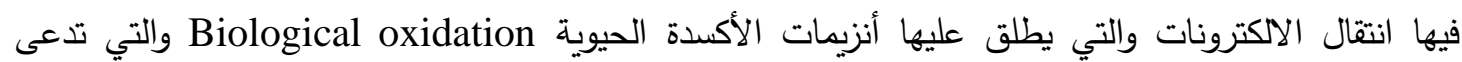
الديهيدروجنيز Dihydrogenase وهي من نوع أنزيمات الأكسدة والاختزال الحاوية على الموقع الفعال المتمثل

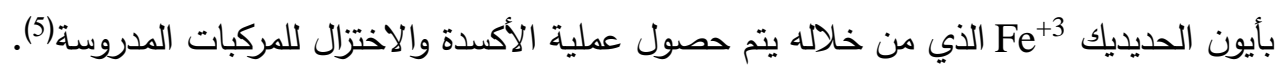
تمت دراسة مسار الفيناثرين عن طريق بكتيريا (Gram Positive Rhodococcus Opacus 1Cp) Nocardioides

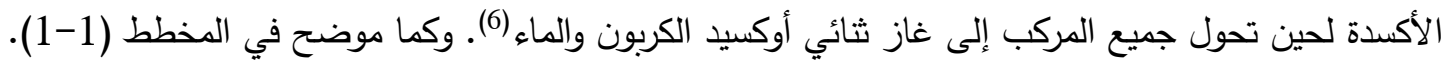

Phenanthrene $\longrightarrow$ 1-Hydroxy-2-Nphthoic acid $\longrightarrow$ (Salicyte route)

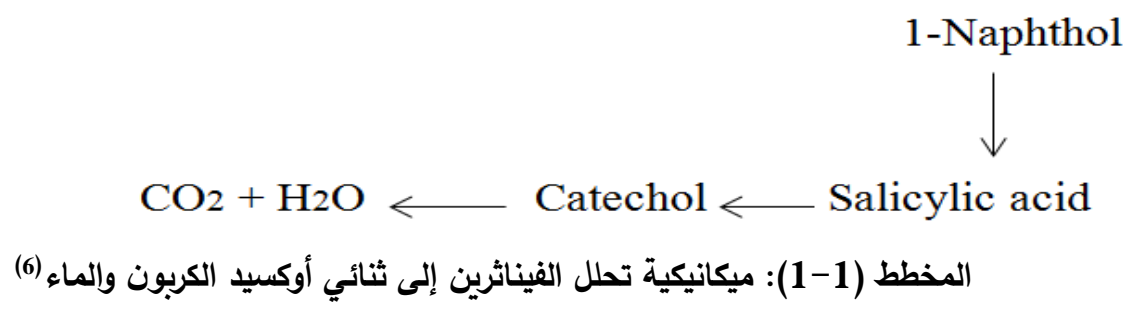

\section{Biodegradetion Constant}

1-3 ثابت سرعة التحلل (التكسير) الحيوي

تعد عملية التحلل الحيوي أو التكسير الحيوي من تفاعلات الرتبة الأولى أو الرتبة الأولى الكاذبة ويمكن تحديد ثابت سرعة التحلل استناداً إلى العلاقة(8,7) :

$-\frac{d c}{d t}=\mathrm{kc} c^{\mathrm{n}}$

$\log \frac{c}{C_{\mathrm{o}}}=k \cdot t$ 
(mg/L) تركيز المادة بعد فتره من الزمن:Co

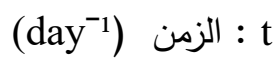

(day $\left.{ }^{-1}\right)(\mathrm{PAH})$ : ثابت سرعة تحلل المادن : : n : رتبة التفاعل :

Log $\frac{c}{C_{o}}$ ويمكن الحصول على قيمة kنابت سرعة التحلل الحيوي) عن طريق رسم العلاقة بين

مقابل الزمن(9).

وتعد عملية التحلل عملية فزيوكيميائية تحدث في التربة ومياه الانهار والبحار أضافة إلى إنها تحدث

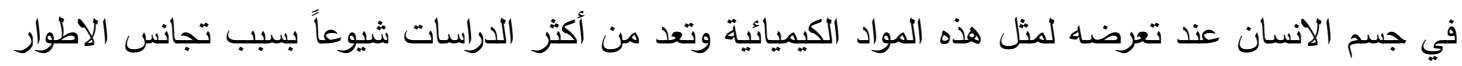
(طور التربة الصلب) مع المادة الهيدروكربونية الصلبة.

وتعتمد عملية إزالة هذه المركبات (تحللها) على جهد التأين للمركب أضافة إلى إن دراسة حركية هذه

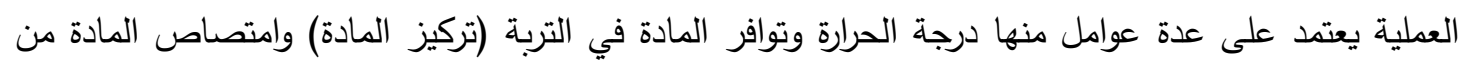
قبل التربة وعملية التهوية (وجود مادة الاوكسجين) وتوفر البكتيريا المحلة الحاوية على أنزيمات الأكسدة

\section{Docking Calculation}

1-4 تثكيل المركب على الانزيم

تعد طريقة دراسة تشكيل (تحميل) المركب على البروتين أو الأنزيم من الدراسات الحسابية والنظرية

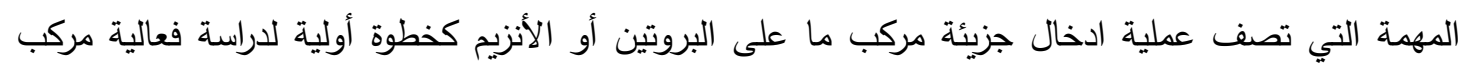

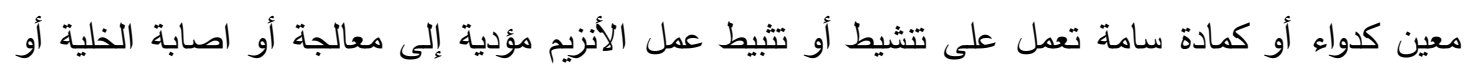

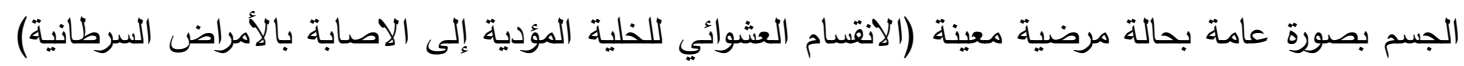

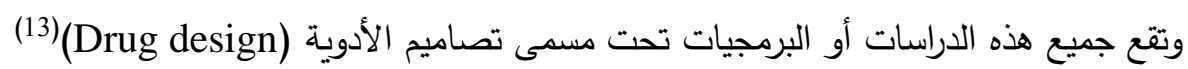

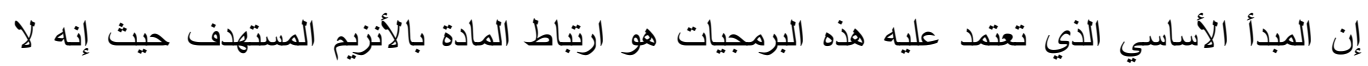

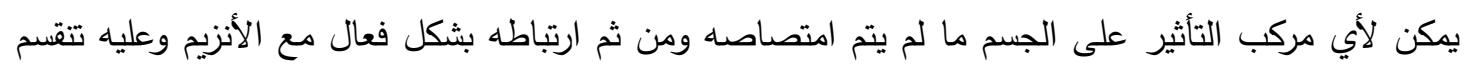
البرمجيات من حيث المبدأ إلى قسمين اساسيين الأول يعتمد على حساب الخصائص الفيزيائية والكيميائية والثاني يعتمد على حساب الخواص السمية والذوبانية في الماء وسرعة الامتصاص في الامعاء والتوزيع في الام لئي

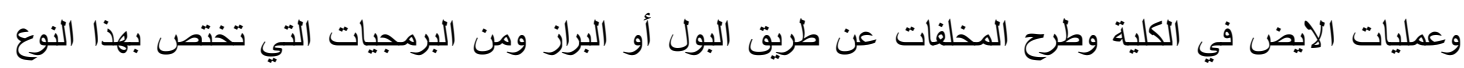

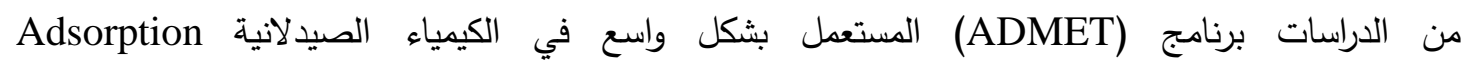
Docking Distribution Metabolism Excertion and Toxcicty الكيمياء وعلوم الحياة أضافة إلى استعماله في الكيمياء الصيدلانية.

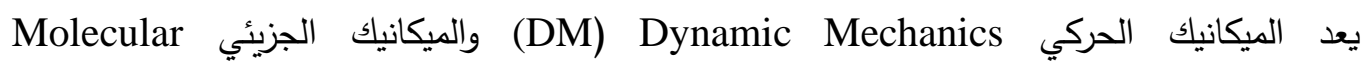
القاعدة الأساسية التي يعتمد عليها هذا النوع من البرمجيات أضافة إلى ميكانيك الكم

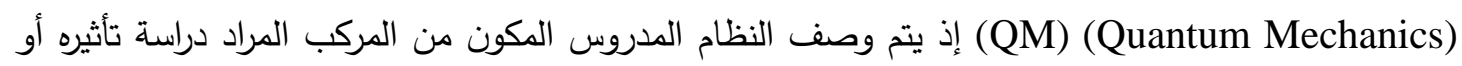
فعاليته على أنزيم معين عن طريق حساب طاقة المجال للجزيئة (Molecular Force Field) والتي تعتمد

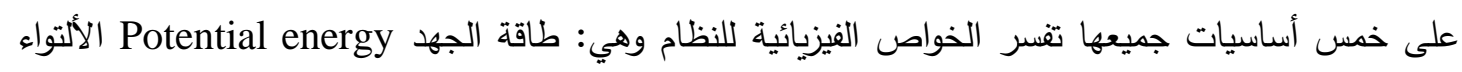
وتشكيل الأواصر Bond geometry الكهروستاتيكية Electrostatic terms وجهد لينارد 
تم تطوير طريقتين لحساب تثكيل (تحميل) المركب على الأنزيم الطريقة الأولى تعتمد على كون الأنزيخ

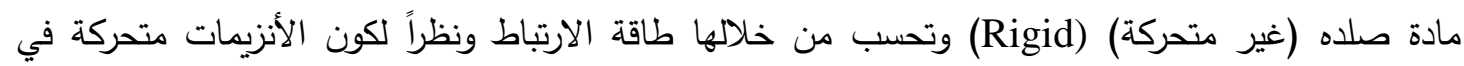
الجسم الحي Flexable فقد تم التوصل إلى طريقة اقرب للواقع وذات نتائج أكثر دقة من خلال استعمال برمجيات متطورة تعتمد على تحريك الأنزيم في المحيط المائي بالاتجاهات الثلاثة ونتيجة لهذه الحركة تتغلغل

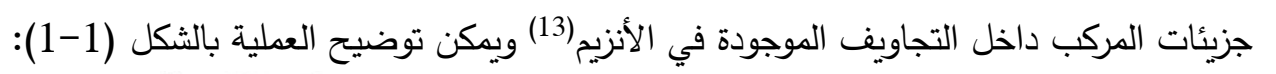
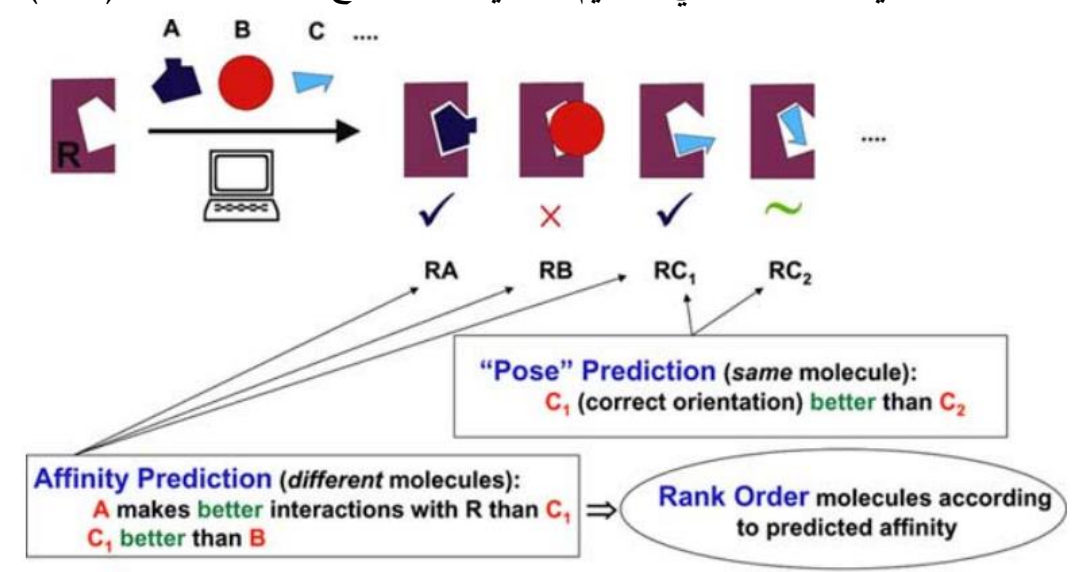

Scoring Function: Interaction Energies, Solvation/Desolvation, Entropy, ....

الثكل (1-1): يوضح ارتباط الأنزيم بالمادة (Receptor) - R(R, - وتمثل المستقبل), اما C,B,A - تمثل جزيئات صغيرة يتم تثبيتها على جزيئة المستقبل (18).

في عام (2014) قام الباحث شارما بدراسة عملية ارتباط الأنزيم البكتيري (3HGI) و (2XSR) من

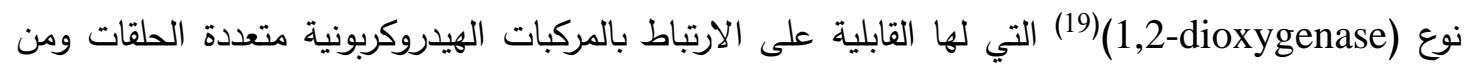
خلال هذه الدراسة التي اجريت على مركبي Benzo[a]pyrene و

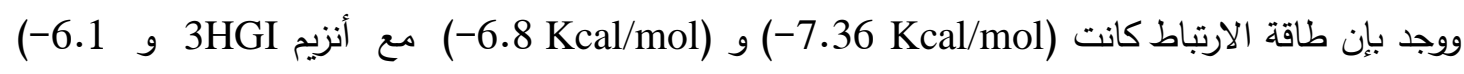
و Kcal/mol) (Dioxygenase يرتبط بشكل أفضل من مركب Chrysene (Benzo[a]pyrene) في حين إن المركب الاخير كان (Catechol 1,2-dioxygenese) (3HGI) [Rhodococcus opacus])

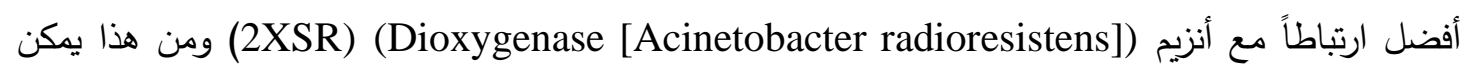
تحديد الأنزيم المستعمل لتحليل مادة معينة أو معرفة تأثيرات المركبات المختلفة على الأنزيم.

\section{Binding energy $\left(\Delta G^{\circ}\right)$}

(20) 5-1

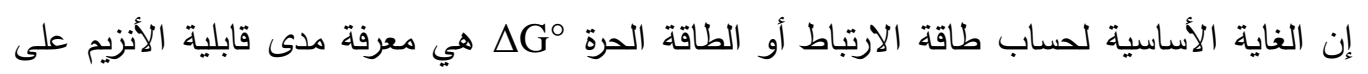

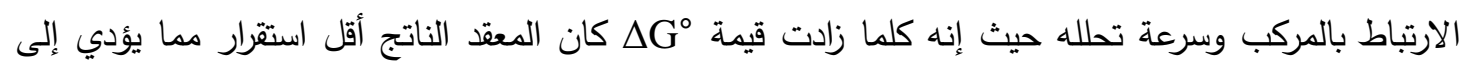
حدوث عملية التحلل بفترة زمنية أقل مقارنة بالمركبات الاخرى ذات طاقات الارتباط القليلة. تمثل طاقة الارتباط بالطاقة الحرة لجبس التي يمكن حسابها باستعمال العلاقة التالية :

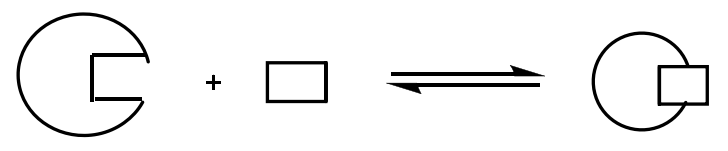


$\mathrm{R}+\mathrm{L} \underset{\mathrm{k}_{\mathrm{d}}}{\stackrel{\mathrm{k}_{\mathrm{a}}}{\rightleftharpoons}} \mathrm{RL}$

$\Delta \mathrm{G}^{\circ}=-\mathrm{R} \mathrm{T} \ln \mathrm{K}_{\mathrm{a}}$

$\mathrm{k}_{\mathrm{a}}=\mathrm{k}_{\mathrm{d}}{ }^{-1}=\frac{[R L]}{[R][L]}$

$\Delta \mathrm{G}^{\circ}=\Delta \mathrm{H}^{\circ}-\mathrm{T} \Delta \mathrm{S}^{\circ}$

من اهم العوامل المؤثرة على قيمة طاقة الارتباط $\Delta \mathrm{J}^{\circ}$ في مثل هذا النوع من الحسابات هي:

1. القوى الداخلية (Inter molecular forces)

Bond تكون هذه القوى قوى ترابطية أو تآصرية يمكن تحديدها من خلال قياس اطوال الأواصر والزوايا . lengths, Bond angles, Dihydral angles

2. القوى الخارجية (Intra molecular forces)

وتكون هذه القوى غير ترابطية وتمثل التداخلات الأكتروستاتيكية والفية التداخلات القطبية Polar interactions والأواصر الهيدروجينية H-bonding تداخلات فاندرفالز , الهيدروفوبية (الكارهة للماء) أو الدهنية (hydro phobicity)

يمكن التأكد من دقة نتائج الطاقة الحرة (طاقة الارتباط) الحسوبة بلدة بعد اجراء عملية تشكيل (تحميل

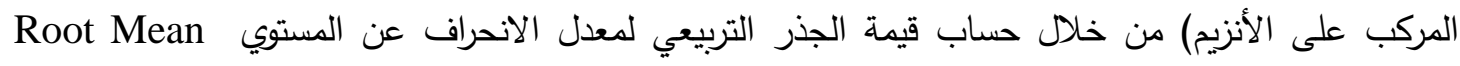
(RMSD) Squared Deviation $\mathrm{RMSD}=\sqrt{\frac{1}{N} \sum_{i=1}^{N}\left(X_{c i}-X_{d i}\right)^{2}+\left(y_{c i}-y_{d i}\right)^{2}+\left(Z_{c i}-Z_{d i}\right)^{2}}$

N = Ci , di

أفضل النتائج يتم الحصول عليها من عملية (Docking) في مثل هذا النوع من الحسابات هي عندما تكون قيمة RMSD اقل من (2.0A) والتي تمثل انحراف الآصرة عن المستوى للمركب المدروس مقارنة بالقيم

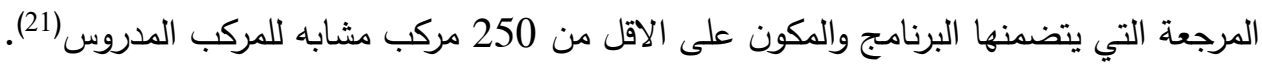

\section{Theoretical Calculation}

2- 2 - 2

تتضمن الحسابات النظرية اختيار أحد عشر مركباً من المركبات الاروماتية متعددة الحلقات والموضحة صيغها وقيم ثوابت سرع التحلل الحيوي لها في الجدول (2-1)(22) والتي تمت دراستهات بطريقة الارومانية عملية تضمنت استخدام بكتيريا تسمى Gram Positive Rhodococcus opacus منتجة لأنزيم كاتيكول 2,1- ثنائي

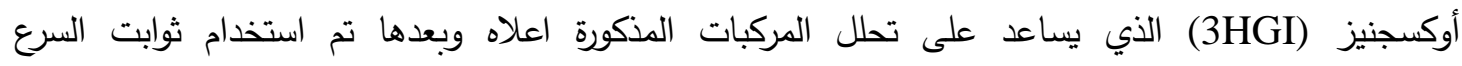
المستحصله كعامل معتمد مقابل طاقة الارتباط المحسوبة نظرياً باستعمال برمجيات فيزوحياتية.

الجدول (2-1): الأسماء والصيغ التركيبية وقيم ثوابت سرعة التحلل الحيوي للمركبات الاروماتية متعددة الحلقات (22)

\begin{tabular}{|c|c|c|c|c|c|}
\hline الرقم ل & اسم المركب & الرمز & الصيغة التركيبية & $\begin{array}{c}\text { Biodeg } \\
\text { Rate } \\
\mathrm{K}_{\mathrm{b}}\left[\text { day }^{-1}\right]\end{array}$ & $\begin{array}{c}\text { Molecular } \\
\text { weight }\end{array}$ \\
\hline
\end{tabular}




\begin{tabular}{|c|c|c|c|c|c|}
\hline 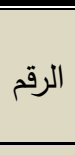 & اسم المركب & 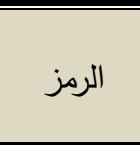 & الصيغة التركيبية & $\begin{array}{c}\text { Biodeg } \\
\text { Rate } \\
\mathrm{K}_{\mathrm{b}}\left[\text { day }^{-1}\right] \\
\end{array}$ & $\begin{array}{c}\text { Molecular } \\
\text { weight }\end{array}$ \\
\hline 1 & Benzene & BEN & & 0.1 & 78 \\
\hline 2 & Acenaphthylene & $\mathrm{ACY}$ & & 0.02 & 152.20 \\
\hline 3 & Phenathrene & PHN & & 0.0447 & 178.2 \\
\hline 4 & Anthracene & ANTH & & 0.0052 & 178.2 \\
\hline 5 & Fluoranthene & FLN & & 0.0018 & 202.26 \\
\hline 6 & Pyrene & PYR & & 0.0027 & 202.3 \\
\hline 7 & Benzo [a] anthracene & $\mathrm{BaA}$ & & 0.0026 & 228.29 \\
\hline 8 & Chrysene & CHR & & 0.0019 & 228.3 \\
\hline 9 & $\begin{array}{l}\text { Benzo [b] } \\
\text { fluroanthene }\end{array}$ & $\mathrm{BbFN}$ & & 0.0024 & 252.3 \\
\hline 10 & $\begin{array}{c}\text { Benzo }[\mathrm{k}] \\
\text { fluroanthene }\end{array}$ & $\mathrm{BkFN}$ & & 0.0005 & 252.3 \\
\hline 11 & $\begin{array}{c}\text { Dibenzo[a,h]anthrace } \\
\text { ne }\end{array}$ & DBahA & & 0.0019 & 278.35 \\
\hline
\end{tabular}

1-2 طريقة حساب طاقة الارتباط مع الانزيم Calculation Method of Energy Bonding with Enzyme

هناك عدة برمجيات تختص بدراسة تشكيل (تحميل) المركب على الأنزيم أو ما يسمى بعملية (Docking) عن الارتباط بين المركب والأنزيم المستهدف وتختلف مسميات البرامج وكذلك سهولة أو صعوبة استعمالها ومنها

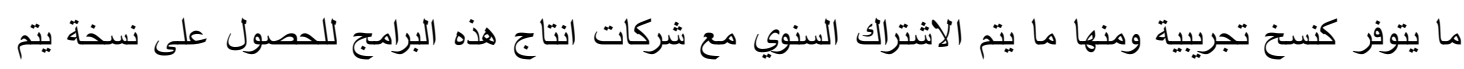

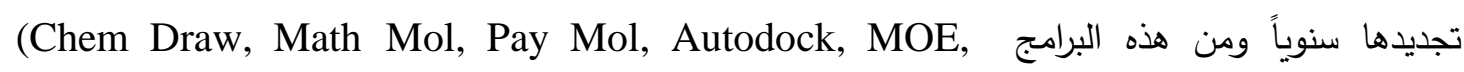
ويحتاج العمل في هذه البرامج على حاسبات متطورة ذات سعة خزن عالية ومعالج بمواصفات Swissdock) خاصة.

في هذه الدراسة تم استعمال برنامج Molecular Operating Enviroment) (2015) MOE). 


$$
\text { تتضمن خطوات العمل على هذا النوع من البرامج ما يلي: }
$$

رسم المركب على الأنزيم واجراء عملية خفض الطاقة (Minimazation) ثم حساب الثحنات واطوال الأواصر والزويا. وتجهيز الأنزيم المستهدف من خلال استدعاءه اما من اقراص خاصة حاوية على الأنزيمات

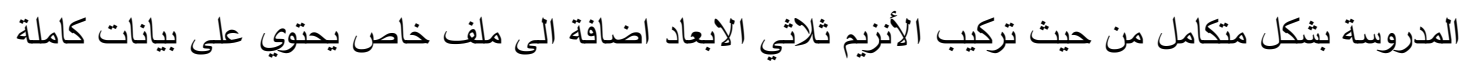
عن الأنزيم من حيث اطوال الأواصر والزوايا المستحصلة من قياس حيود الاشعة السينية للأنزيم أو إن يتم لإنيات

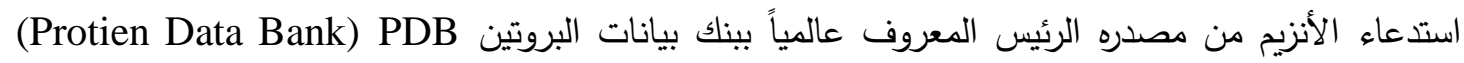
وفي هذه الدراسة استعمل انزيم بكتيري من نوع كاتيكول 2,1 - ثنائي اوكسجنيز - Catechol 1,2 Gram Positive Rhodococcus والذي يرمز له بـ (3HGI والمستخلص من بكتيريا dioxygenase

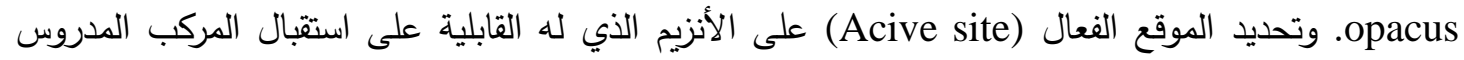
والتآصر معه. وفي هذه الدراسة تم تحديد ايون الحديديك (Fe+3) كموفع فعال لحصول عملية الأكسدة(18). واستعمل ايعاز Dock الموجود ضمن البرامج التي من خلالها يتم اجراء أكثر من مئة (100) محاولة يتغير فيها موقع المركب على طول سلسلة الأنزيم للحصول على أفضل موقع ارتباط وبطاقة أقل ما يمكن بحيث تتراوح

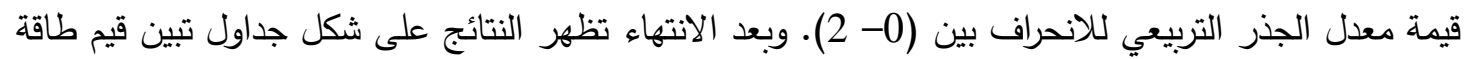
الارتباط ومعدل الجذر التربيعي للانحراف. وبعد الحصول على النتائج يتم استعمال بعض الايعازات للحصول

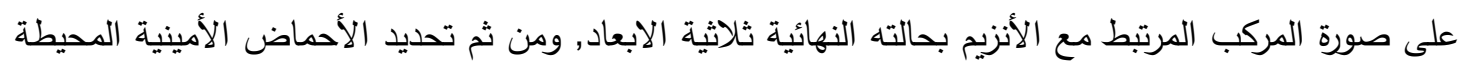
وطبيعة تآصرها مع المركب.

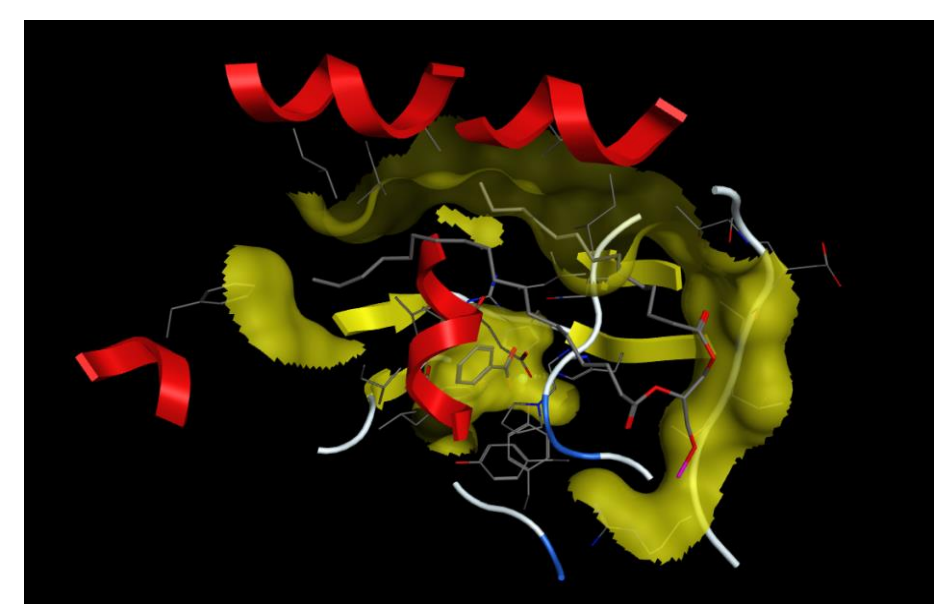

الثكل رقم (2-1) : شكل ثلاثي الأبعاد لأنزيم كاتيكول 2,1 - ثنائي أوكسجنيز 3HGI

\section{Results and Discussion}

3

تستعمل الكائنات الحية الدقيقة مثل البكتيريا كمعالجات حيوية بايولوجية لتحطيم الجزيئات الهيدروكربونية متعددة الحلقة PAHs نظراً لاحتوائها على أنزيمات لها القابلية على أكسدة هذا النوع من بن بائية المركبات وتحويلها إلى جزيئات أوكسجينية صغيرة ويعد أنزيم كاتيكول 2,1 - ثنائي أوكسنيز أنزيم 1,2-dioxygenase البكتيري وهو أنزيم مؤكسد - مختزل ينتج من قبل نوع من البكتيريا يطلق عليهات Gram Positive Rhodococcus opacus الأوكسجين مكوناً مركبات أوكسجينية وكاتيكولات. 
من خلال البيانات المستحصل عليها من قبل بنك المعلومات للبروتين PDB تبين بأن أنزيم مكون من سلسلة واحدة مكونة من 280 حامض أميني (Chain A) يحتوي على اربع مواقع فعالة وهي ايون

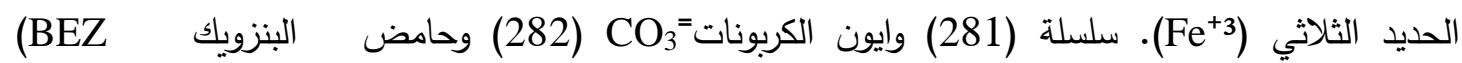
4-hydroxy, N,N,N-Trimethyl, (284) 6PL ( $\left(\mathrm{C}_{42} \mathrm{H}_{85} \mathrm{O}_{8} \mathrm{P}\right) \quad$ ومركب (283) $\left.\mathrm{C}_{7} \mathrm{H}_{6} \mathrm{O}_{2}\right)$ OXO-7-[palmitoyloxy (methyl)]-3,5,8TrioxA-4-phosphahexacosan-1-Aminium 4-

(13) وتعد هذه المواقع مستقبلة للمركبات الداخلة على الأنزيم سواء كانت مركبات منشطة أو مثبطة oxide

\section{Calculation of Bonding Energy}

-3-3 - حساب طاقة الارتباط

تعد عملية التحلل الحيوي للمركبات الهيدروكربونية متعددة الحلقة من العمليات المهمة التي تعمل على تخلص البيئة منها والتي تعد ملوثات بيئية خطيرة لكونها مواد سامة وتعد عملية التحلل الأنزيمي احدى الطرائق التي يتم من خلالها التخلص من هذا النوع من المركبات.

في هذه الدراسة تم حساب طاقة الارتباط (الطاقة الحرة) لأحد عشر مركباً من المركبات الهيدروكربونية

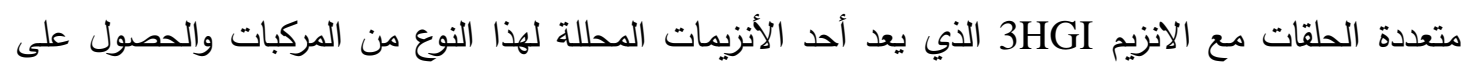
نتائج لقيم طاقة الارتباط تتوافق نوعاً ما مع قيم ثوابت سرع التحلل الحيوي لعدد من المركبات المدروسة. الجدول رقم (3-1) يبين قيم ثوابت السرعة وطاقة الارتباط ومعامل الجذر التربيعي للانحراف والأحماض لتوات الأمينية

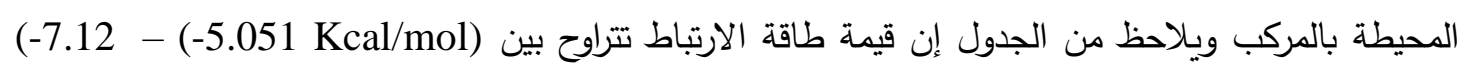

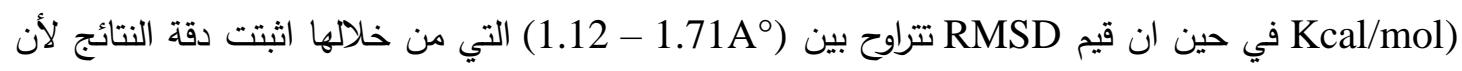
هذه القيم يفضل إن تكون أقل من (2Aº 1 استتاداً الى دراسات سابقة (20). تبين من خلال هذه الدراسة بإن طاقة الارتباط تتناسب عكسياً مع ثوابت سرعة التحلل الحيوي وبمعامل

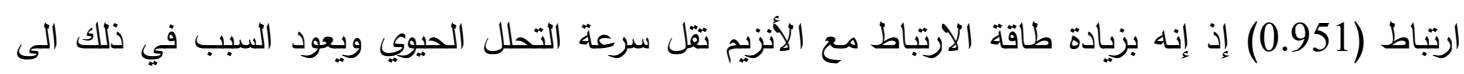

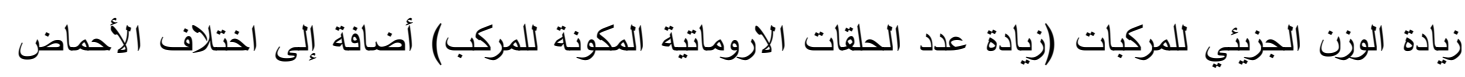
الأمينية المحيطة بالمركب والمساعدة على تحلل هذه المركبات. إن اعلى قيمة لثابت سرعة التحلل الحيوي تمثلت بمركب البنزين (احادي الحلقة) يقابها أقل قيمة لطاقة الارتباط (5.051 Kcal/mol-) في حين إن أقل قيمة لثابت التحلل للمركب رقم (10) يقابلها قيمة طاقة ارتباط

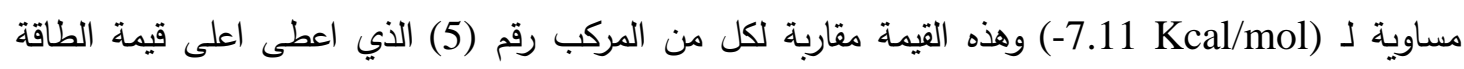

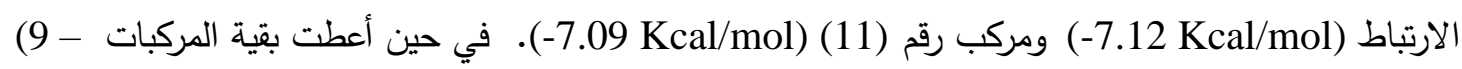

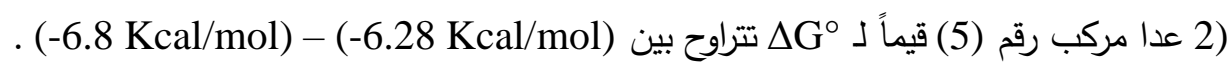

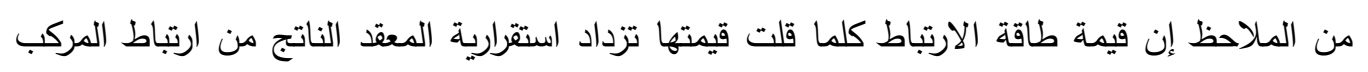

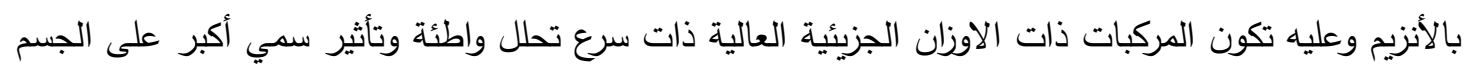

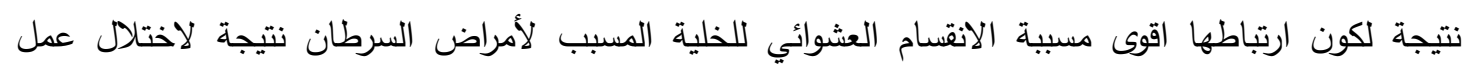
الأنزيم والمركبات الناتجة عن عملية التحلل.

الجدول (3-1): قيم ثوابت سرعة التحلل الحيوي وطاقة الارتباطومعدل الجذر التربيعي للانحراف RMSD والاحماض الامينية المحيطة بالمركبات 
إيجاد العلاقة بين ثوابت سرعة التحلل البايولوجي وبعض الصفات الفيزياوية المحسوبة نظرياً لعدد من المركبات ...

\begin{tabular}{|c|c|c|c|c|}
\hline $\begin{array}{l}\text { Comp. } \\
\text { No. }\end{array}$ & $\begin{array}{c}\mathrm{k}_{\mathrm{b}} \\
\left(\mathrm{day}^{-1}\right)\end{array}$ & $\begin{array}{c}\Delta \mathrm{G}^{\circ} \\
\mathrm{Kcal} / \mathrm{mol}\end{array}$ & RMSD & Amino acid \\
\hline 1 & 0.1 & -5.051 & 1.12 & $\begin{array}{lll}\operatorname{Arg}(217), \quad \operatorname{Val}(81), & \begin{array}{l}\operatorname{Pro}(105), \\
\operatorname{Tyr}(106),\end{array} & \operatorname{Tyr}(196),(104)\end{array}$ \\
\hline 2 & 0.02 & -6.32 & 1.34 & $\begin{array}{lll}\text { Phe(165), } & \text { Pro(199), } & \begin{array}{l}\text { Gln(197), } \\
\text { Gly(160), } \operatorname{Tyr}(106)\end{array}\end{array}$ \\
\hline 3 & 0.0447 & -6.28 & 1.25 & $\begin{array}{lll}\operatorname{Pro}(199), \quad \operatorname{Phe}(165), & \begin{array}{l}\mathrm{G} \ln (197), \\
\operatorname{Tyr}(161), \operatorname{Tyr}(106)\end{array} \\
\end{array}$ \\
\hline 4 & 0.0052 & -6.66 & 1.63 & $\begin{array}{lll}\text { Pro(199), Phe(165), } & \begin{array}{l}\text { Gln(197), Gly(160), } \\
\text { Tyr(106), Ile(198) }\end{array}\end{array}$ \\
\hline 5 & 0.0018 & -7.12 & 1.41 & $\begin{array}{llll}\text { Pro(199), Phe(165), } \begin{array}{c}\operatorname{Tyr}(161), \\
\text { Gly(160), } \operatorname{Tyr}(106),\end{array} \\
\end{array}$ \\
\hline 6 & 0.0027 & -6.68 & 1.72 & $\operatorname{Pro}(199), \quad \operatorname{Tyr}(161), \quad \operatorname{Gln}(197), \quad \operatorname{Gly}(106)$ \\
\hline 7 & 0.0026 & -6.71 & 1.68 & $\begin{array}{lccc}\text { Asp(201), } & \operatorname{Thr}(200), & \operatorname{Phe}(155), & \operatorname{Pro}(199), \\
\text { Ile(198), } & \text { Gln(197), } & \operatorname{Tyr}(161), & \operatorname{Tyr}(106), \\
& & \text { Gly(160) }\end{array}$ \\
\hline 8 & 0.0019 & -6.8 & 1.34 & $\begin{array}{lccc}\text { Asp(201), } & \text { Phe(155), } & \text { Pro(199), } & \operatorname{Ile}(198), \\
\text { Gly(180), } & \text { Tyr(200), } & \text { Gln(197), } & \operatorname{Tyr}(161), \\
\operatorname{Tyr}(106)\end{array}$ \\
\hline 9 & 0.0024 & -6.77 & 1.64 & $\begin{array}{llll}\operatorname{Asp}(201), & \operatorname{Phe}(155), & \operatorname{Pro}(199), & \operatorname{Tyr}(106), \\
\operatorname{Tyr}(161), & \operatorname{Gln}(197), & \operatorname{Gly}(160), & \operatorname{Tyr}(200)\end{array}$ \\
\hline 10 & 0.0005 & -7.11 & 1.28 & $\begin{array}{llll}\operatorname{Asp}(201), & \operatorname{Pro}(199), & \operatorname{Phe}(155), & \operatorname{Tyr}(106), \\
\operatorname{Tyr}(161), & \operatorname{Tyr}(200), & \operatorname{Gln}(197), & \operatorname{Gly}(160)\end{array}$ \\
\hline 11 & 0.0019 & -7.09 & 1.53 & 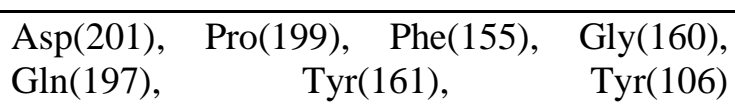 \\
\hline
\end{tabular}

ويلاحظ من الجدول ايضاً إن الأحماض الأمينية التي تحيط بالمركب عادة ما ترتبط به بآواصر هيدروجينية أو تداخلات هيدروفوبية من نوع $\pi$ - $\pi(23)$.

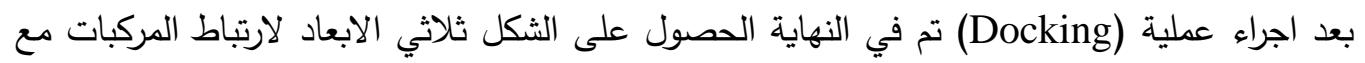

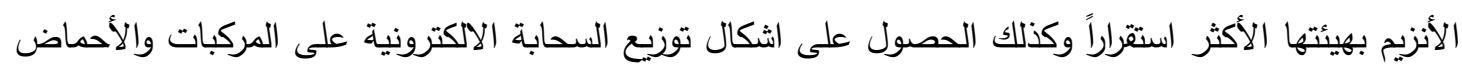
الأمينية المحيطة بها والتي تأخذ مواقع حول جزيئة المركب المدروس استتاداً إلى صفاتها كأحماض أمينية حامضية, قاعدية, هيدروفوبية (قطبية أو غير قطبية) وكما موضح في الاشكال المدرجة في الجدول (3-2). الجدول (3-2): مواقع ارتباط المركبات الاروماتية متعددة الحلقات والأحماض الأمينية المحيطة بها في سلسلة الأنزيم 
ريان بشير محمود \& زاهدة احمد نجم

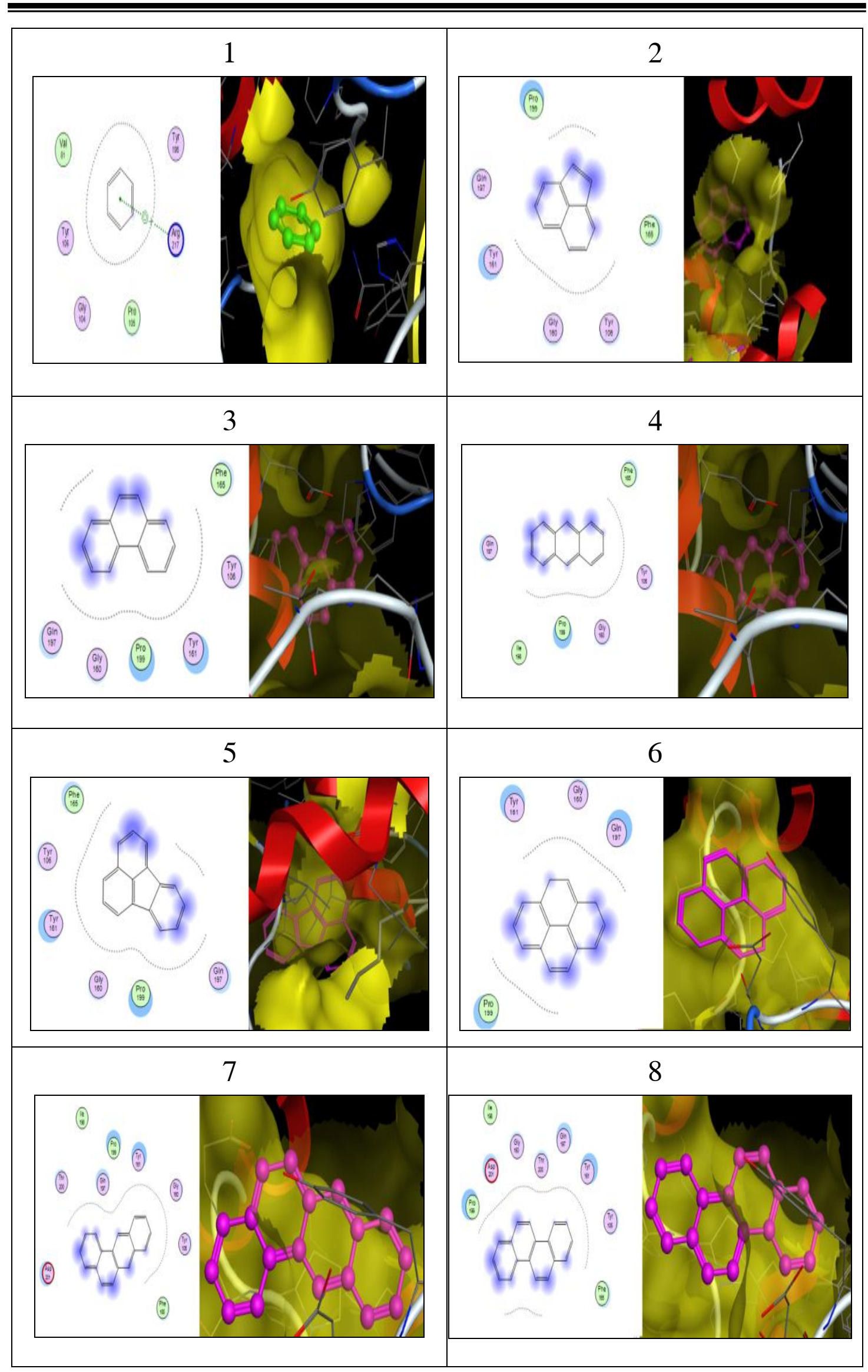


إيجاد العلاقة بين ثوابت سرعة التحلل البايولوجي وبعض الصفات الفيزياوية المحسوبة نظرياً لعدد من المركبات ...

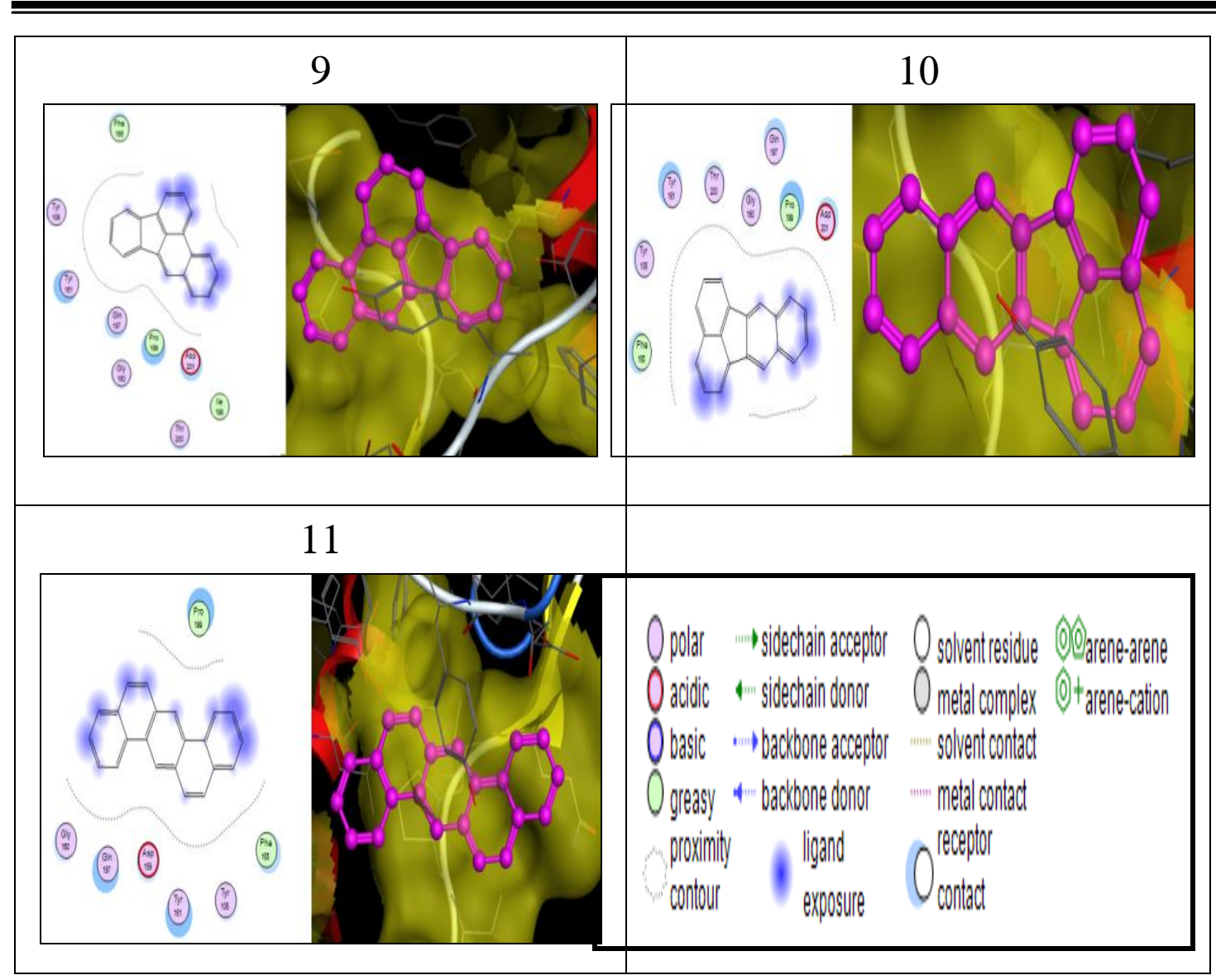

المصادر

1) Langston J.W., O’Hara S., Pope D.N., Davey M., Shortridge E., Imaumura M., Harino H.A., Kim H.C. and Vane, "Bioaccumulation surveillance in Milford Haven waterway", Environmentol Monitoring and Assessment. 184(1): 289-3 ISSN 0167-6369 (2011).

Doi: 10.1007/s 10661-011-. \$1968-z.

2) Dipple A., "Polycyclic Aromatic Hydrocarbon Carcinogenesis". Polycyclic Hydrocarbon and Carcinogenesis. ACS Symposium series. 283. American Chemical ISBN. 0-8412-0924-3.17-1pp society (1985).

3) Hassouny Jadoua Abdallah, Desertification, Degradation of the ecosystem, Dar Degla Publishing \& Distribution, Jordan, 1fr ed., PP86, (2010).

4) Kingdom of Saudi Arabia, Ministry of Education Imam Mohammed bin Saud Islamic University, Standing Committee for the Prevention of Rediation and Environmental Pollution.

5) Tarek Younes Ahmed and Louay Abd Ali Hilali, Biochemistry, University of Mosul, Ibn Al-Ather Publishing House, (2010).

6) Iwabuchi T. and Harayama S., "Biochemical and genetic characterization of carboxybenzaldehyde dehydrogenase, an enzyme involved in phenanthrene degradation by Nocardioides sp". Strain KP7. J. Bact. 179: 6488 - 6494 (1997). 
7) Alexander M., "Biodegradation and Bioremediation", Academic Press, Inc., San Diego, CA(1999).

8) Kwon SH., Kim JH, Cho D., "An analysis method for degradation kinetics of lowly concentrated PAH solutions under UV light and ultrasonication". Journal of Industrial and Engineering Chemistry. ;15(2):157-62(2009).

9) Yang Y., Shu L., Wang X., Xing B., Tao S., "Effects of composition and domain arrangement of biopolymer components of soil organic matter on the bioavailability of phenanthrene". Environmental Science and Technology. 2010;44(9):3339-44(2010).

10) Tayssir Kadri, "Tarek Rouissi Research center for Eco-Enviromental sciences", Chinese Academy of sciences. Vol. 2017 page 52-74, (2016).

11) Nilanjana D., Preethy C., "Microbial degradation of petroleum hydrocarbon contaminants: An overview. Biotechnology Research International". (2010), (2011).

12) Henkel M., Müller MM., Kügler JH., Lovaglio RB., Contiero J., Syldatk C. et al., "Rhamnolipds as biosurfactants from renewabie resources: Concepts for nextgeneration rhamnolipid production". Process Biochemistry. (2012).

13) TIP Rev. Esp. Cienc. Quin. Biol. Vol. 21, supl. 1 (2018).

14) Rebert E. Notar., "Pharmacokinetics and Molecular Modification Implications in drug design and evaluation", Journal of pharmaceutical sciences, Vol. 62, Issue 6, page 865-881(1973).

15) Puzgn T., et. al., "Recent Advaces QSAR Studies 283-304 (2010).

16) Iopes P.E.M., Guvench O., \& Mackerell A.D., "Current Status of Protein Force Fiells For Molecular Dynamics Simulations". Methods in Molecuar Biology, 1215, 47-71(2015) .

17) Monticell and Tieleman, "force fielcls for classical molecular dynamics methods in molecular bology", 924, 197-213(2013).

18) Structure-Based Drug Design Docking and Scoring, 8, 312-328(2007).

19) Romano Kroemer T., Sharma T., "Journal of Chemical and Pharmaceutical Research" 6(6): 873-877(2014).

20) Thomas funkhouser, "Review: Protein-ligand Docking Methods", Princeton university Cs 597(2005).

21) Waszkowycz, Clark \& Gancia, Wiley Interdisciplinary Reviews; computation Molecular science 1, 12, 229-259.

22) Catherine A. Peters, Christopher D. Knightes and Derick G. Brown, "Long-Term Composition Dynamics of PAH-Containing NAPLs and Implications for Risk Assessment", Environ. Sci. Technol. 33, 4499-4507(1999).

23) Kamil kuca, Daniel Tun, Molecular 23:1103, (2018). 